\title{
Using Framing as a Lens to Understand Context Effects on Expert Reasoning
}

\author{
Tara Slominski, ${ }^{\dagger}$ Andrew Fugleberg, ${ }^{\dagger}$ Warren M. Christensen, ${ }^{\ddagger}$ John B. Buncher, ${ }^{\ddagger}$ \\ and Jennifer L. Momsen ** \\ †Department of Biological Science and ₹Department of Physics, North Dakota State University, \\ Fargo, ND 58108-6050
}

\begin{abstract}
National calls to transform undergraduate classrooms highlight the increasingly interdisciplinary nature of science, technology, engineering, and mathematics (STEM). As biologists, we use principles from chemistry and physics to make sense of the natural world. One might assume that scientists, regardless of discipline, use similar principles, resources, and reasoning to explain crosscutting phenomena. However, the context of complex natural systems can profoundly impact the knowledge activated. In this study, we used the theoretical lens of framing to explore how experts from different disciplines reasoned about a crosscutting phenomenon. Using interviews conducted with faculty $(n=10)$ in biology, physics, and engineering, we used isomorphic tasks to explore the impact of item context features (i.e., blood or water) on how faculty framed and reasoned about fluid dynamics, a crosscutting concept. While faculty were internally consistent in their reasoning across prompts, biology experts framed fluid dynamics problems differently than experts in physics and engineering and, as a result, used different principles and resources to reach different conclusions. These results have several implications for undergraduate learners who encounter these cross-disciplinary topics in all of their STEM courses. If each curriculum expects students to develop different reasoning strategies, students may struggle to build a coherent, transferable understanding of crosscutting phenomena.
\end{abstract}

\section{INTRODUCTION}

Undergraduate students pursuing a science, technology, engineering, and mathematics (STEM) degree typically spend a great deal of their first 2 years navigating a busy schedule full of introductory science courses, including physics, chemistry, math, and biology. In these courses, students are introduced to the principles, concepts, and skills that comprise the fundamentals of these disciplines, many of which are interdisciplinary or crosscutting concepts that transcend a single domain or introductory science course (National Research Council [NRC], 2012).

Unfortunately, the inherent disciplinary segregation of higher education leaves little opportunity for instructional collaboration across disciplines. As a result, STEM faculty rarely know how these crosscutting concepts are taught in disciplines outside their own. Without this pedagogical content knowledge, how do we know whether students are prepared to use and apply crosscutting concepts in biology courses? In fact, through interdisciplinary conversations, we discovered that introductory physics and human anatomy and physiology (HA\&P) courses at our institution broach the crosscutting concept of fluid dynamics in fundamentally different ways, and thus have quite different expectations for our students. Further, our experiences mirrored the perspectives of Redish and Cooke (2013), who described the experiences and insights gained through a multiyear curricular collaboration between a biologist and physicist. In their essay, Redish and Cooke (2013) described distinct epistemological and cultural differences between their respective disciplines as a means to explain instructional discrepancies relating to crosscutting concepts such as fluid dynamics. While

Kimberly Tanner, Monitoring Editor Submitted Nov 15, 2019; Revised Jun 26, 2020; Accepted Jun 29, 2020

CBE Life Sci Educ September 1, 2020 19:ar48 DOI:10.1187/cbe.19-11-0230

*Address correspondence to: Jennifer L. Momsen (jmomsen@gmail.com).

() 2020 T. Slominski et al. CBE-Life Sciences Education () 2020 The American Society for Cell Biology. This article is distributed by The American Society for Cell Biology under license from the author(s). It is available to the public under an Attribution-Noncommercial-Share Alike 3.0 Unported Creative Commons License (http://creativecommons.org/licenses/ by-nc-sa/3.0)

"ASCB®" and "The American Society for Cell Biology ${ }^{\circledR}$ " are registered trademarks of The American Society for Cell Biology. 
these disciplinary characterizations align with our experiences teaching foundational fluid dynamics, our present study seeks to add empirical evidence to support these disciplinary differences. Popular introductory textbooks from physics and HA\&P revealed even more disparate takes on foundational fluid dynamics (Silverthorn, 2009; Knight et al., 2010; Giordano, 2013; Martini et al., 2015).

We opted to focus our research on the crosscutting concept of fluid dynamics, specifically the factors that impact fluid movement in a system. In introductory physics courses, fluid dynamics is often covered over several weeks toward the end of the first semester of a two-semester sequence. Students in these courses range from first-year physics and engineering majors to upper-division students in other disciplines that require a two-semester physics sequence (biology, chemistry, exercise science, etc.). Understanding fluids and fluid dynamics relies on students' previous mastery of concepts like statics, dynamics, work, and energy - in particular, these concepts are integral to understanding the structure of the equations that describe fluid dynamics. Introductory physics students are expected to relate these fundamental concepts of forces and energy to understand the fundamental equations governing fluids. In addition, students must also apply these equations and concepts to many different physical systems to make predictions about fluid behavior under a variety of conditions. In contrast to HA\&P courses (see next paragraph), not all physics courses or textbooks at this level include a section dealing with viscosity and its related concept, resistance.

In physiology courses, including HA\&P, fluid dynamics is essential to understanding the cardiovascular system and the urinary system. Emphasis in HA\&P courses is typically placed on the impacts of fluid dynamics on blood pressure and water regulation. The Human Anatomy and Physiological Society has recognized the importance of fluid dynamics to HA\&P content by articulating multiple learning objectives pertaining to blood flow and urine formation (Human Anatomy and Physiology Society, 2019). In alignment with these learning objectives, HA\&P students are typically expected to use the fundamental equations and principles of fluid dynamics to understand and make predictions regarding hemodynamics and urine formation under varying conditions.

Taken together, it appears that our physics and HA\&P courses train students to use different reasoning approaches for fluid dynamics problems. For this research, we define reasoning as the activation, accumulation, and coordination of multiple cognitive resources (e.g. conceptual resources, epistemological resources, and affective factors) in an attempt to solve or make sense of a problem or scenario (diSessa 1993; Hammer and Elby, 2003; Hammer et al., 2005; Redish and Kuo, 2015). In effect, our courses train students to develop separate reasoning approaches for introductory physics and HA\&P. This observation is troubling: Many students enroll simultaneously in these courses, and because instruction in HA\&P and physics is so distinct, students would be ill-served even trying to transfer ideas across courses. In response to this, we developed a research project to 1) explore how STEM experts (physics, biology, and engineering) could reason about foundational fluid dynamics so differently and 2) determine whether item surface context impacted expert reasoning about foundational fluid dynamics.

\section{Crosscutting Concepts}

A tacit component of undergraduate education is that students develop the ability to work fluently across domains of science and to understand and use crosscutting concepts when problem solving (NRC, 2012; McDonald, 2015). However, students struggle to transfer their understanding within a discipline, let alone across disciplinary boundaries (NRC, 2000). Students often compartmentalize their courses and, by extension, science disciplines: Energy in physics is "not the same" as energy in chemistry or biology, and different rules or ways of reasoning may apply.

Experts, however, appear to have little trouble identifying and using crosscutting scientific concepts to solve complex problems in science. Indeed, research documents that novices and experts have different approaches to problem solving (Smith and Good, 1984; Camacho and Good, 1989; Simmons and Lunetta, 1993; Bodner and Herron, 2002). Chi and colleague's (1981) foundational study found, through a simple sorting task, that novices tended to sort physics problems according to surface features of the problem, while experts sorted problems based on underlying physics principles. Recent work in biology has documented a similar phenomenon: Novice learners in biology tend to sort tasks by superficial features, while experts used core biological concepts (Smith et al., 2013). The present work aims to extend the novice-expert literature by examining whether experts in different disciplines (i.e., biology, physics/engineering) use similar reasoning and concepts when answering fluid dynamic problems.

Little research has explored the similarities and differences in how crosscutting concepts are taught across disciplines, which may impact students' ability to transfer their understanding and subsequently build connections. Experts have deep content knowledge, which includes these crosscutting concepts. However, discipline norms, ways of thinking, and epistemological disciplinary differences (Redish and Cooke 2013) may cause experts to teach about crosscutting concepts in narrow ways that inhibit students' transfer abilities. We argue that, to understand how students are taught to reason with and about crosscutting concepts, it is critical to understand how experts teaching these courses think about crosscutting concepts. To explore experts' reasoning across disciplines, we introduce a theoretical frame from cognitive science: framing and resources.

\section{Theoretical Framework: Framing and Resources}

Under the traditional view of student difficulties research, "alternative conceptions" or "misconceptions" are incorrect answers resulting from strongly held, context-specific, stable, and incorrect knowledge constructs (Smith et al., 1994; Hammer, 1996; Maskiewicz and Lineback, 2013). An alternative view is that wrong answers can be the result of activating and integrating smaller ideas that are not necessarily inherently wrong but are used in ways not appropriately suited for the case at hand (diSessa, 1993; Hammer et al., 2005). In this alternative theoretical framework, framing and resources, student thinking is not stable, but dynamic and context dependent (Gouvea and Simon, 2018).

The theoretical framework of framing and resources specifically attempts to model a perspective of cognition as emergent and dynamic by identifying specific cognitive or procedural resources, which are fine-grain ideas that may be consciously or 
subconsciously activated by an individual (Hammer and Elby, 2003; Hammer et al., 2005). Developed by Hammer and Elby (2003), with contributions by Redish and Scherr (Hammer et al., 2005), this framework builds from diSessa's knowledgein-pieces framework $(1993,1988)$. The framing and resources theoretical framework has been used extensively in physics education research. Studies using this framework span physics and science instruction across K-12 (e.g., Elby and Hammer, 2010; Berland and Hammer, 2012) and at the university level (e.g., Hammer, 2000; Wittmann and Black, 2015). When attempting to name resources, investigations most often involve qualitative case studies of single individuals (e.g., Lising and Elby, 2005; Farlow et al., 2019) or small groups of subjects (e.g., Bing and Redish, 2008; Loverude, 2015), and rarely from free-response data (e.g., Goodhew et al., 2019). In biology education research, this more dynamic view of student cognition is not yet widespread, but has recently been used as a lens to explore student difficulties widely recognized as misconceptions (Gouvea and Simon, 2018). The framing and resources theoretical framework has been recognized as a valuable tool for explaining students' intuitive ideas about human physiology (Slominski et al., 2017).

Within this theoretical framework, the specific resources that an individual activates depend on how the individual frames, often subconsciously, a particular situation. An individual's framing could depend on features within the problem itself, such as the phrasing of the question, the syntax, the notation used, the context or setting in which the problem was given, or who was asking the particular question (Chi et al., 1981; diSessa et al., 2004; Hammer et al., 2005; Sabella and Redish, 2007; Nehm and Ha, 2011; Gouvea et al., 2019). In contrast to the misconceptions view of student difficulties, the framing and resources theoretical framework incorporates the impact context features can have on cognition and, ultimately, the answers an individual provides.

When applying the framing and resources theoretical framework, it is critical to note that the resources activated by an individual are heavily influenced by how they have framed the particular problem. It is possible that an individual has additional resources that might be activated by a different problem or task and that an alternative framing, or a shift to a different frame, might activate some of these additional resources. While resources are often associated with a particular frame (or frames), it is important to recognize resources are not exclusive to a particular frame. Rather, it is possible resources can be activated in different combinations in different frames. Therefore, analysis of responses can never conclude that an individual does not have a particular resource, rather analysis can only conclude that an individual did not activate a resource. It is quite possible that an individual could have activated a particular resource if the individual had framed the problem differently.

\section{Context and Reasoning}

A hallmark of this dynamic view of cognition is that framing and resource activation happen in the early moments of an individual encountering a problem or scenario. Because framing is a situated event, the conditions surrounding the problem or scenario greatly impact the resulting frame the individual employs (Smith et al., 1994; Hammer et al., 2005; Gouvea and Simon, 2018). As an individual begins the process of problem solving, the context of the problem may cause the learner to subconsciously (or at times consciously) frame the problem (e.g., this is a math problem or this is a biology problem) and therefore impact the suite of concepts and problem-solving resources the learner activates. For example, biology education researchers have found that student reasoning about evolution is profoundly impacted by item context. Nehm and Ha (2011) suggested that students approach and reason differently about evolution involving trait gain versus trait loss. Similarly, Heredia and colleagues (2012) found that students were more likely to reason correctly about natural selection when discussing animals than plants. In addition, students were more likely to apply inaccurate "survival of the fittest" reasoning when the assessment contained an "unfriendly" animal. Collectively, these studies demonstrate that seemingly insignificant context features of an assessment cause students to retrieve different knowledge and to subsequently reason differently.

Experts have a well-developed set of discipline-specific resources and associated frames that allow them to reason well in multiple contexts within their own disciplines. What is unclear is the extent to which these frames and resources are changed when experts are presented with a problem outside their own disciplines. Additionally, it is not clear that experts from different disciplines would use identical (or similar) frames and resources on the same crosscutting concept. If experts from different disciplines use different frames and resources when explaining the same crosscutting concept, this may impede students from building connections across disciplines on these crosscutting concepts.

For this research, we are particularly interested in the role of experts' disciplinary expertise in how they frame a problem about a crosscutting science concept and how context intersects with that framing. Based on our interdisciplinary conversations surrounding fluid dynamics, we knew experts in HA\&P and physics talk about this crosscutting concept differently, and we wished to understand why. Thus, we explored the role of disciplinary expertise on expert reasoning through the theoretical framework of framing and resources. We also investigated how contextual surface features intersected with framing to impact experts' reasoning approaches. Specifically, we asked:

1. To what extent do STEM experts from physics, biology, and engineering differ in their reasoning about foundational fluid dynamics?

2. Does context impact expert framing and subsequent reasoning about foundational fluid dynamics?

\section{METHODOLOGY \\ Study Participants}

This research took place at North Dakota State University, a midwestern land-grant university with high research activity (Carnegie Classification of Institutions of Higher Education, n.d.). To attract experts with diverse disciplinary backgrounds, we solicited faculty participants from biology, physics, and engineering departments. Potential interview participants were identified through review of their respective research biographies and courses taught posted on the university website. We sought out faculty with either research or teaching experience in 1) animal physiology, 2) agricultural irrigation, or 3) general 


\section{Biology Version (BV Prompt)}

The figure below shows three different blood vessels (A, B, and C) with blood flowing through them (designated by the gray arrow on the left). The volume of blood entering the left end of the blood vessel every second is the same in Systems A, B, and $C$. The pressure in the blood is the same at points $D, E$, and $F$. The blood viscosity is very low. The diameter on the left end of each blood vessel is the same $(5 \mathrm{~cm})$.
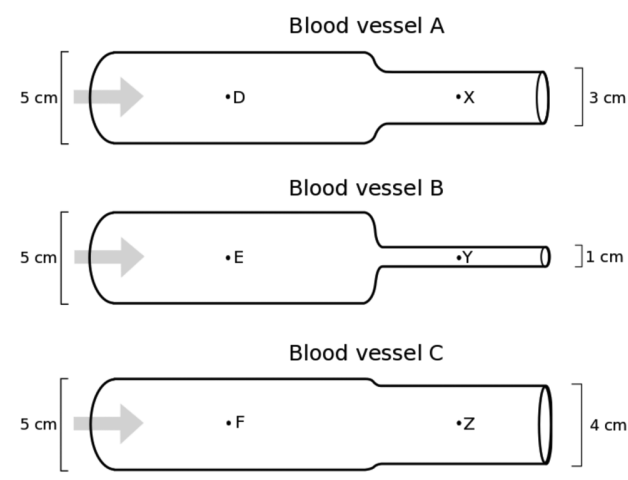

"Order the speed of the blood coming out of the right end of the blood vessels A, B, and C. If two or more blood vessels have equal speeds at the exit, say they are equal. Please try to think aloud."

"Order the fluid flow rate coming out of the right end of blood vessels $\mathrm{A}, \mathrm{B}$, and $\mathrm{C}$. If two or more blood vessels have equal fluid flow rates, say they are equal. Again, try to articulate your thinking as you answer this question."

"Order the pressure of the blood in the blood vessels at points $X$, $Y$, and $Z$. If two points have equal pressures, say they are equal. Please try to think aloud."

"Order the resistance in the blood at points $X, Y$, and $Z$. If two points have equal resistances, say they are equal. Please try to think aloud."

\section{Non-Biology Version (WP Prompt)}

The figure below shows three different pipes (A, B, and C) with water flowing through them (designated by the gray arrow on the left). The volume of water entering the left end of the pipe every second is the same in Systems A, B, and C. The pressure in the pipe is the same at points $D, E$, and $F$. The water viscosity is very low. The diameter on the left end of each pipe is the same $(5 \mathrm{~cm})$.
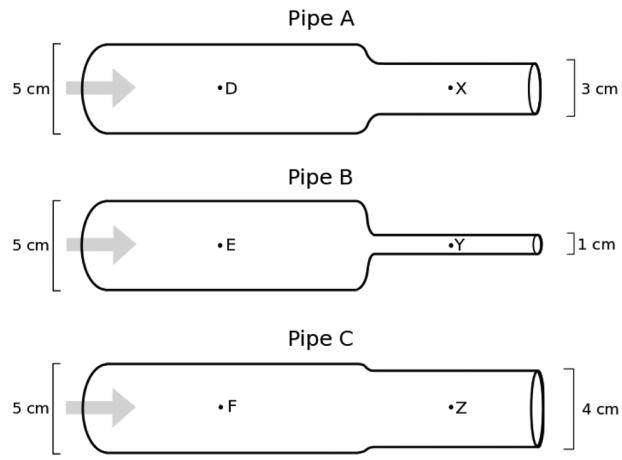

"Order the speed of the water coming out of the right end of the pipes A, B, and C. If two pipes have equal speeds at the exit, say they are equal. Please try to think aloud."

"Order the fluid flow rate (volume of water flowing per unit of time) coming out of the right end of pipes A, B, and C. If two pipes have equal fluid flow rates, say they are equal. Again, try to articulate your thinking as you answer this question."

"Order the pressure of the water in the pipes at points $X, Y$, and $Z$. If two points have equal pressures, say they are equal. Please try to think aloud."

"Order the resistance in the water at points $X, Y$, and $Z$. If two points have equal resistances, say they are equal. Please try to think aloud."

FIGURE 1. The biology version (BV) and non-biology version (WP) of our isomorphic prompt.

fluid dynamics. Twelve candidates were identified and contacted via email. Of those, 10 faculty agreed to participate in our study, four from biology (Blake, Bernie, Bailey, Blair), three from physics (Pacey, Peyton, Pat), and three from engineering (Emerson, Emery, Ellis). All interview participants had PhDs in relevant disciplines and were not involved in the prompt development described in the next section. Throughout the article, to avoid implicit gender biases, we assume these pseudonyms are nongendered and subsequently adopt the personal pronouns "they," "them," and "their."

All interviews were conducted by the same interviewer (T.S. graduate student) in May of 2017. This interview structure created a potential power imbalance: A graduate student interviewed a faculty member. This power imbalance may have impacted the dynamics of the conversations that took place during the interviews. For example, faculty may have adopted the role of a teacher when talking to a graduate student rather than engaging in collegial discussions.

The interviewer was known to the biology participants and one physics participant (Pat), but unknown to the remaining interview subjects. All participants had a range of experience teaching the underlying concept of fluid flow in the undergraduate setting, and all were actively engaged in research.

\section{Prompt Development}

Given our goal of determining how different contextual features could affect experts' reasoning approaches about foundational fluid dynamics, specifically at the introductory level, we designed two isomorphic prompts pertaining to fluid dynamics (Figure 1). The biology version of the prompt was situated in the context of blood and blood vessels (we refer to this as the "BV" prompt) and the non-biology version was situated in the context of water and pipes (we refer to this as the "WP" prompt). The prompts contained identical figures and text, with the exception that "blood" and "blood vessel(s)" were used in the BV prompt where "water" and "pipe(s)" were used in the WP prompt.

Isolating context effects on reasoning approaches is challenging and places several demands on prompt construction. First, we needed to create a prompt that could be turned into 
two isomorphic prompts-that is, identical except for context-related language (e.g., exchanging water for blood, pipes for vessels). Such a prompt enables comparisons of how item surface features impact participants' reasoning in the most direct manner possible. Second, both prompt versions needed to be feasibly answered by all of our experts and could not contain technical terms specific to a discipline. This constraint reduces ambiguity in what is cueing respondents' ideas and focuses on the surface features of the prompt itself. As such, words like "compliance" (more likely to be encountered in a biology course), and "incompressible" (more likely to be encountered in a physics course) were not used in either version of the prompt. Additionally, words not typically encountered in both courses at the introductory level (such as "gradient" in an introductory physics course) were also omitted. Finally, to further our ultimate goal of understanding context effects on students' reasoning about crosscutting concepts, we created a prompt that would be appropriate for either an introductory physics or HA\&P course.

To achieve these goals, our prompt was coconstructed by a biologist (T.S.) and physicist (J.B.B.) with review and feedback from W.M.C. and J.L.M. and additional experts in biology and physics. We also solicited feedback from experts in engineering, in part because earlier conversations with engineering colleagues in our STEM education community at NDSU revealed that engineers might think about fluid flow in ways that merged ideas from biologists and physicists. This makes intuitive sense: physicists often reason about foundational, idealized systems, while biologists reason about living-and often messy-systems. Engineers work at a nexus of idealized and real systems, providing a sort of middle ground between physicists and biologists. The prompt was then piloted with faculty from all three disciplines (biology, physics, and engineering). Their responses were used to create a revised version of the prompt that we use in the current research.

The prompt asks participants to rank the fluid speed, fluid flow rate, pressure, and resistance at three locations (X, Y, and $\mathrm{Z}$ ) in three different systems (A, B, and C, respectively; Figure 1) and explain the reasoning behind their rankings. The order of items was deliberate. We asked about fluid speed first, because, based on our pilot studies, it seemed likely that participants would be most familiar with this concept. We next asked about fluid flow rate, as this concept is central to reasoning about cardiovascular physiology, physics, and engineering. Third, we asked about pressure, another core concept in cardiovascular physiology, which makes use of the Bernoulli equation, a fundamental concept in physics and engineering. We asked about resistance last, because we discovered during prompt development that this concept was most likely to provide disparate responses across disciplines.

We believe it is essential to note that we were not attempting to assess expert reasoning of a phenomenon from their respective disciplines and were not, as a result, focused on the "correctness" of experts' responses. Instead, we were primarily concerned with the reasoning experts used to rank the various concepts. We do, however, provide samples of "expected" or "normative reasoning" in the fields of physics and biology in the Supplemental Material. These lines of reasoning reflect how we would expect experts to reason within the contexts of their discipline.

\section{Interview Protocol}

After participants provided their ranking and reasoning for a particular concept, we then asked them to provide a definition of the concept (e.g., fluid flow rate) they were working with, and whether their answer was consistent with their definition. If participants provided an equation, they were asked to recall where they learned that equation. If participants provided an analogy, they were asked to describe what caused them to think of that analogy. However, for the fourth and final question, which dealt with resistance, the protocol differed slightly. If participants could not provide a definition of resistance, they were asked for their best guess at the question when hearing the word "resistance" in this context. After an initial guess, if the interviewer was asked for a definition, participants were told that "some biologists would say resistance is 'a force that opposes movement"' and were asked to answer the question using that definition.

We used a semistructured interview protocol, recording audio and video for all interviews. All interviews were transcribed using Express Scribe. Participants responded first to the prompts considered outside their disciplines: biology faculty first reasoned about the WP prompt, while physics and engineering faculty first reasoned about the BV prompt. Using this approach, we intentionally did not cue participants to their disciplinary knowledge. Participants then completed a distractor task, meant to redirect the participant to think about a concept unrelated to fluid dynamics, before responding to the prompts within their disciplines. This ordering of prompts supported our efforts to determine whether context cues impacted reasoning or whether participants would adopt a frame that differed from the context presented in the prompt.

\section{Data Analysis}

We used thematic analysis (Braun and Clarke, 2006) to identify broad themes in expert reasoning about fluid dynamics, a crosscutting concept. From these themes, we then propose potential frames. This inductive approach involved three phases of analysis, briefly described in the following sections.

Analysis Phase 1: Initial Reading. Phase 1 of our analysis was completed by T.S. and A.F. We began by independently reading the responses to the speed question from all 10 participants and making notes of early thoughts on faculty responses. During this initial reading, we independently identified key words or phrases that seemed to exemplify a participant's reasoning. We came together and compared notes, identifying potential or early themes in responses to the speed question. We repeated this process for the fluid flow rate question. After individually reading all 10 responses and taking notes, we compared the early themes from the speed question to those of the fluid flow rate question and noticed there were both similarities and differences in the initial themes. We repeated this process with the pressure question and the resistance question, each time reflecting back to initial themes identified in the previous responses.

Analysis Phase 2: Generalizing Themes. Phase 2 of our analysis was completed by T.S. and A.F., followed by discussion with J.L.M. We compared themes gathered from reading all four questions embedded in our interview prompt and combined 
TABLE 1. Rankings provided by experts in response to the blood and vessels (BV) prompt and the water and pipes (WP) prompt ${ }^{\mathrm{a}}$

\begin{tabular}{|c|c|c|c|c|c|c|c|c|}
\hline & \multicolumn{4}{|c|}{ Out-of-discipline context } & \multicolumn{4}{|c|}{ Discipline context } \\
\hline & Speed & FFR $^{\mathrm{b}}$ & Pressure & Resistance & Speed & FFR $^{\mathrm{b}}$ & Pressure & Resistance \\
\hline Biologists & \multicolumn{4}{|c|}{ WP } & \multicolumn{4}{|c|}{ BV } \\
\hline Bernie & BAC & $=$ & BAC & BAC & BAC & $=$ & BAC & BAC \\
\hline Bailey & BAC & $\mathrm{CAB}$ & $\mathrm{BAC}^{\mathrm{c}}$ & BAC & BAC & $\mathrm{CAB}$ & $={ }^{\mathrm{c}}$ & BAC \\
\hline Blair & BAC & BAC & BAC & BAC & BAC & BAC & BAC & BAC \\
\hline Peyton & BAC & $=$ & $=$ & BAC & BAC & $=$ & $=$ & BAC \\
\hline Pat & BAC & $=$ & BAC & BAC & BAC & $=$ & BAC & BAC \\
\hline Engineers & \multicolumn{4}{|c|}{ BV } & \multicolumn{4}{|c|}{ WP } \\
\hline Emerson & BAC & $=$ & BAC & BAC & BAC & $=$ & BAC & BAC \\
\hline Emery & BAC & $=$ & $\mathrm{CAB}$ & BAC & BAC & $=$ & $\mathrm{CAB}$ & BAC \\
\hline
\end{tabular}

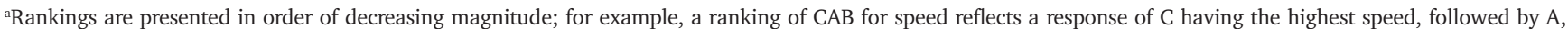
followed by B.

bFFR, fluid flow rate.

'Calls attention to an instance where the participant's answer is not consistent across prompts.

${ }^{\mathrm{d}} \mathrm{cnr}$, could not respond; Pacey stated they were unable to provide an answer based on the information provided.

themes that reflected similar ideas. When themes were disproportionately represented across a single question or discipline, we questioned the utility of that theme and dropped themes when appropriate. We generated a list of themes based on the early themes that emerged from initial readings. We formalized descriptions of these themes and generated an early coding rubric.

We independently reread all 10 responses to the speed question, identifying the presence of early themes and documenting specific examples of dialogue that aligned with theme descriptions. We compared themes and the accompanying dialogue. Any disagreements were discussed until a consensus was reached. We then updated our rubric. We repeated this process with the three remaining questions. Each iteration resulted in minor theme modifications and refinement.

Analysis Phase 3: Finalizing Themes. Phase 3 of our analysis was completed by T.S. and A.F., with discussion with J.L.M. We came together and discussed patterns in our data and lingering questions. After consulting with J.L.M., we further refined the theme descriptions, and one theme was removed, leaving us with our final rubric consisting of four themes: Switching Context, Disciplinary Knowledge, Everyday Knowledge, and Relationships and Equations. We again independently read all 10 transcripts and coded the dialogue according to the final rubric. We met one final time to discuss any disagreements, reaching resolution in all instances.

Thematic analysis serves as a critical step toward identifying potential frames from which participants might pull specific resources. The dialogue captured by a given theme served as our evidence to make a claim that experts adopted a given frame in response to our interview prompt. In some cases, a theme maps directly to a particular frame, as was the case for our Disciplinary Knowledge theme. In other cases, a theme reflects evidence that an expert moved between frames (e.g., Switching Context).
Finally, we note that we are not, in the present work, identifying resources. While it is possible that an analysis of a single individual from our data set or an analysis of the data set on the whole could produce specific named resources, we questioned the utility of identifying resources from experts (a practice that is not yet present in the literature). Instead, we decided the far more salient results from our data were the framing and subsequent reasoning of our interviewees. Research from physics education, the STEM discipline where the theoretical framework of framing and resources has the strongest adoption, has yet to establish a common methodology for identifying conceptual resources. Researchers who have named conceptual resources have typically done so through a series of qualitative investigations specifically designed to elucidate evidence of student use of those resources or to focus on the resources of a single interviewee (e.g., Farlow et al., 2019). The grain size and evidence for what constitutes a named resource, therefore, can vary greatly, from those investigators seeking to identify the very smallest possible distinct piece of conceptual knowledge resource, such as diSessa's phenomenological primitives (diSessa, 1988), to much larger conceptual- or mathematical-level resources. Our intent here is not to identify resources but to describe the potential frames used by experts from different disciplines and, by extension, the impact of framing on expert reasoning.

\section{RESULTS AND DISCUSSION \\ Comparing Rank-Order Predictions}

In this section, we focus our analysis on the specific rankings experts provided, and we discuss how those rankings differ across prompt versions and disciplinary background. Comparing the rankings experts provided in response to our prompts illuminates the initial differences in expert reasoning.

When reporting rankings, we present them in order of decreasing magnitude. For example, a ranking of $\mathrm{CAB}$ for the speed question means the subject gave a response with $\mathrm{C}$ having 
the largest speed, and B as the lowest. For every direct quote, we represent the prompt version and subquestion in brackets (e.g., [BV, pressure] or [WP, resistance]). We believe it is crucial to make clear the context to which the participant was responding, as our argument centers on the context of the prompt and how the participant responds to that context.

\section{Comparisons of Predictions within a Discipline}

In most cases, faculty within a discipline generated similar rank-order predictions about both contexts (BV, WP; Table 1), although there were a few notable exceptions. Among the biologists, the most disagreement was observed in responses to the fluid flow rate item, with two biologists providing a CAB ranking (Table 1), one biologist a BAC ranking, and one biologist a ranking of all equal.

In contrast, among physicists and engineers, the most disagreement was observed in response to the pressure items in both contexts (BV, WP; Table 1). Three participants provided a BAC ranking, two participants a ranking of all equal, and one participant a ranking of $\mathrm{CAB}$. In addition, Ellis, an engineer, consistently differed from all other physicists and engineers in their rankings of all items across contexts. It is interesting to note that Ellis has arguably the most extensive research experience in fluid dynamics of all our study participants. Characterization and measurement of fluid flow is one of Ellis's self-identified research areas, and they have experience working in biological systems. Using the theoretical lens of framing, we might argue that Ellis has framed this problem differently than the other engineering and physics faculty, and even biology faculty. Considering Ellis has a great deal of experience working with fluids in a biological setting, it is possible that Ellis's knowledge and experience of biological systems has impacted the way they frame this problem and thus the conceptual resources activated.

\section{Comparisons of Predictions within Individuals}

We observed three instances in which faculty changed their answers when given the second, within-discipline prompt. Bailey initially provided the ranking of BAC when reasoning about pressure in response to the WP prompt, but switched to all equal when responding to the BV prompt. When asked to explain their reasoning about pressure on the BV prompt, Bailey said:

\footnotetext{
"This is just like me guessing, purely based on intuition. But the idea is like if you have blood pressure, you know you're measuring that, usually you can measure it at one point in the body, and it's assumed that it's the same everywhere else. I know that that's not a great assumption, but that's like how medicine passes forward, right?" [BV, pressure]
}

Based on this response, Bailey may have been drawing on ideas of mean arterial pressure and peripheral resistance, originating from their knowledge of cardiovascular dynamics. Bailey is a clear example of biologists consistently framing these questions in a biological way.

We also observed what may be a context effect in Pacey's response to the pressure questions. When presented with the BV version of the prompt, Pacey struggled to generate a response based on the constraints of our prompt:
"So ... You know, I'm struggling with this. I don't know what you ... You have to tell me what you mean by pressure exactly, then I can try to answer the question. Right now I'm really puzzled, right? I don't know how to rank it at the moment." [BV, pressure]

Pacey goes on to explain why this question is problematic for them. They eventually provide a rank order, but immediately reiterate their concerns and, ultimately, state they are unable to provide an answer.

"When you squeeze this, um, fluid through these, um, different tubes here, this small capillary and then this big one here, it has to speed up as I argued, right? So if the speed is connected to the pressure, then that would suggest that the pressure is larger here than it is here [pointing to handout] and, um, in the smallest tube you would have the largest pressure. But again, without defining a pressure properly, I cannot answer the question. So I feel unable to answer it."

Later in the interview, when Pacey is asked about pressure on the WP prompt, they restate their concern regarding the definition of pressure in this problem but are more willing to provide a response to the question. In this case, we see that Pacey may be more comfortable making assumptions about the system when presented with their own disciplinary context.

The third instance of within-subject variation comes from Ellis in response to the resistance question. When presented with the BV version of our prompt, Ellis provided a ranking of CAB. However, on the WP version, they changed their answer to BAC. When asked to explain their reasoning, Ellis said:

\footnotetext{
"Yeah, I was looking at the walls so, so I may have said it wrong before. Now that I think of it this way it would define it ... Define it how much force distance is here, so you have to do, uh ... Basically, this will see less actually, and I think I said it the other way last time, so." [WP, resistance]
}

In this quote, we see Ellis articulate how their approach differed between the two versions of the prompts. In the BV prompt, Ellis was focusing on resistance as a property of the walls of the vessels. When presented with the WP version, they identified resistance as a property of the fluid. As stated earlier, Ellis has experience working with and measuring fluid dynamics in biological systems. It is possible Ellis's robust experience working with a system similar to the one portrayed in the BV prompt may have impacted their approach to solving the BV prompt and may explain why their responses were different across the two versions of the prompt.

\footnotetext{
"Yeah, I see they're both flowing, they both have the same properties. Viscosity is very low. Um, the volume of fluid is the same. See, there is one other thing you do when you try to replicate the bloodstream. You're not gonna use real blood in general, it's difficult. You use something similar and something similar has similar, uh, properties. So in this case, you know, you could tell me the blood viscosity is very low, water viscosity is very low, and we use, actually, water to model blood sometimes."-Ellis
} 


\section{Comparisons of Predictions across Disciplines}

When looking across disciplines, we see much more variation in how experts answered the individual questions embedded in our interview prompts, particularly in responses to the fluid flow rate and pressure questions. The biologists in our study often assigned different rank orders than experts from physics and engineering (Table 1). Engineers typically provided rank orders more similar to those offered by physicists, though there were still multiple instances of disagreement across the two disciplines.

Analysis of rank ordering seems to suggest that experts from biology versus physics and engineering differ in how they conceptualize and reason about fluid dynamics. In the next section, we specifically explore experts' reasoning to determine whether, in fact, experts from different disciplines frame fluid dynamics problems differently.

\section{Comparing Reasoning to Propose Frames}

We present sample excerpts from our interviews (see Table 2) that supplement and expand the examples used within the narrative. Our goal was to share as much of the qualitative data as possible in order to illuminate our thematic analysis. The quotes chosen are indicative of the broader pattern of responses we observed across all interviews.

In the sections that follow, we present our data using the four themes generated through thematic analysis. These themes allow us to organize the patterns we observed in expert responses and support the articulation of our proposed frames. Our interdisciplinary interpretation of the reasoning encapsulated in these four themes led us to propose three frames: a Biological Frame, a Physical Frame, and an Everyday Fluids Frame. We describe how each of the four themes evidence our proposed frames.

\section{Disciplinary Knowledge as Evidence of Framing}

In this section, we present expert reasoning that was representative of the themes Disciplinary Knowledge or Relationships and Equations. We interpreted the themes of Disciplinary Knowledge and Relationships and Equations as evidence of an interview subject operating within a Biological Frame or Physical Frame.

Physicists and engineers frequently used equations and generalizable relationships when generating their predictions. The formal equations were different from the two variable relationships we observed among biologists, in that they involved many quantities and were often named principles or equations. Specifically, both physicists and engineers used variations of the Bernoulli equation:

\footnotetext{
"Yeah, the same, if the same volume per time is entering the left-hand side it's got to come out the other side. Uh, that's Bernoulli's principle, by the way."-Emerson [BV, fluid flow rate]

"Okay. If I'm, if I tried to make sense of what ... um ... So I'm just interested in Bernoulli's principle, it's more like that's how it is."-Pat [BV, pressure]
}

They also used the continuity equation and its expression in terms of fluid speed:

"Oh, okay. The equation, uh ... The original, the basic form is $\mathrm{Q}$ is equal to VA."-Emery [BV, speed]
In contrast to physicists and engineers, biologists used fewer equations when generating their predictions. Instead, biologists would more typically identify a proportional relationship between two variables, in particular how a change in one variable or property would affect another component of the system. For example, Bernie said:

"So the pressure increases as you decrease the diameter because you're trying to push the same amount of water into a smaller space, and so that's going to increase pressure, that's going to increase the force of it pressing against the outsides of the pipe." [WP, pressure]

Comparing the use of relationships and equations across experts, we find disciplinary experts use distinct reasoning strategies. Biologists in our sample were far less likely to employ robust equations, but instead focused on the implications a change in one variable would have on another and on the broader system. In contrast, physicists and engineers relied heavily on equations necessary to quantify the phenomena represented in our prompt. This observation aligns with the cultural and epistemological differences proposed by Redish and Cooke (2013) and indicates experts approach both interview prompts (in- and out-of-context prompts) in a manner similar to how they would approach problems in their disciplines. Connecting this observation to our theoretical framework, our evidence suggests experts have employed their respective discipline-oriented frames (e.g., a Biological Frame or a Physical Frame) in response to both versions of our prompt and have thus activated resources consistent with that disciplinary frame.

Beyond the use of relationships and equations, we found biologists had a tendency to use biological terms like "blood vessel" and "cardiovascular" when asked about the WP prompt, indicating they are using biological content expertise to reason through the problem. In addition, biologists expanded the boundaries of the system, bringing in ideas from cardiovascular physiology to explain their responses to the WP prompt (Table 2, Disciplinary Knowledge, Blair). For example, Blake used ideas of cardiac output and peripheral vessel resistance to provide a definition of "pressure":

\section{"It's almost like I keep wanting to go back to, well pressure is cardiac output times resistance. Like I just have that ... I don't know. That feeling of needing to, like, rely on that equation." [WP, pressure]}

This focus on cardiac output when asked about the WP prompt suggests Blake is using conceptual resources activated in response to a Biological Frame. By focusing on cardiac output, Blake's response places emphasis on the importance of blood distribution to the tissues and thus indicates their reasoning is (at least partially) informed by the emergent phenomena authentic to the cardiovascular system. This behavior also aligns with the ideas of Redish and Cooke (2013) that highlight the emphasis biologists place on complex and emergent properties of real-world phenomena.

To the biologists, it seems this additional, discipline-specific biology knowledge was helpful for explaining the WP fluids problem, despite the WP prompt containing no explicit reference to a biological system. These experts are activating knowledge 
TABLE 2. Examples of expert responses to the BV and WP prompts

\begin{tabular}{|c|c|c|c|}
\hline & Biology faculty & Physics faculty & Engineering faculty \\
\hline Disciplinary Knowledge & $\begin{array}{l}\text { "Right, because the pressure } \\
\text { would be, shoot it out } \\
\text { further. There's more } \\
\text { pressure. So this one ... one } \\
\text { of the things that's making } \\
\text { me think about ... 'cause I } \\
\text { haven't had physics, but I } \\
\text { have had physiology ... is } \\
\text { how do we do this ... like if } \\
\text { you consider pipe B, where } \\
\text { you have venous flow or } \\
\text { arterial flow and you go } \\
\text { down to small capillaries, } \\
\text { right? And one of the things } \\
\text { you have to have is multiple } \\
\text { outlets for that otherwise } \\
\text { you'd have to blast open your } \\
\text { capillaries." - Blair [WP, } \\
\text { pressure] }\end{array}$ & $\begin{array}{l}\text { "A shear gradient. Viscosity is a } \\
\text { material property that has to do } \\
\text { with the shear gradient. It's not, } \\
\text { it's not a f-Hmm ... Sorry, let me } \\
\text { rephrase that. Loss has to do with } \\
\text { the shear gradient times a } \\
\text { constant that we call viscosity. } \\
\text { Viscosity is a material property so } \\
\text { it's the same for the material, but } \\
\text { the, the loss or the hardness of } \\
\text { pushing the fluid has to do with } \\
\text { how far the walls are apart."-Pey- } \\
\text { ton [BV, resistance] }\end{array}$ & $\begin{array}{l}\text { "We're not going to have a perfect } \\
\text { system. We're going to, uh, have to } \\
\text { pay, pay for the thermodynamic } \\
\text { laws."-Emery [WP, resistance] }\end{array}$ \\
\hline $\begin{array}{l}\text { Disciplinary Knowledge: } \\
\text { relationships and } \\
\text { equations }\end{array}$ & $\begin{array}{l}\text { "Um ... well, 'cause if you were } \\
\text { taking a let's say fixed } \\
\text { volume of fluid and trying to } \\
\text { shove it through a much let's } \\
\text { say uh higher surface area to } \\
\text { volume tube, then it's just } \\
\text { going to exert a lot more } \\
\text { pressure on that tube."-Bai- } \\
\text { ley [WP, pressure] }\end{array}$ & $\begin{array}{l}\text { "So blood is fairly incompressible, I } \\
\text { believe. So since you have the } \\
\text { same current left and right ... That } \\
\text { means material conversion per } \\
\text { unit length along the flowing } \\
\text { direction, meaning that the speed } \\
\text { has to grow when the diameter of } \\
\text { the vessel becomes smaller."- } \\
\text { Pacey [BV, speed] }\end{array}$ & $\begin{array}{l}\text { "Yeah, the same, if the same volume } \\
\text { per time is entering the left-hand } \\
\text { side it's got to come out the other } \\
\text { side. Uh, that's Bernoulli's } \\
\text { principle, by the way."-Emerson } \\
\text { [BV, fluid flow rate] }\end{array}$ \\
\hline Switching Context & $\begin{array}{l}\text { "Or that how fast blood flows. } \\
\text { So when you said speed, the } \\
\text { first thing that came to my } \\
\text { mind was actually just blood } \\
\text { flow. I didn't think about it in } \\
\text { any other way besides blood } \\
\text { flow."-Blake [WP, speed] }\end{array}$ & $\begin{array}{l}\text { "Flow rate... I don't know how you } \\
\text { define it. It could be ... It could } \\
\text { mean it's the velocity per, um, } \\
\text { particle that is moving with the } \\
\text { flow. But it could also mean it's the } \\
\text { total amount of fluid that is } \\
\text { passing at a given point per unit } \\
\text { time."-Pacey [BV, fluid flow rate] }\end{array}$ & $\begin{array}{l}\text { "Well, you've got the same volume or } \\
\text { flow rate, so many gallons per } \\
\text { minute or whatever, right? And } \\
\text { you've got same pressure, pressure } \\
\text { hasn't changed. So you've got a } \\
\text { smaller area, right? So, flow in } \\
\text { pipes is related to ... if you've got } \\
\text { the same flow coming in to all of } \\
\text { these and you got the same flow } \\
\text { going through here [pointing to } \\
\text { handout], the only way you can } \\
\text { make up for it is increase the } \\
\text { velocity."-Emerson [BV, speed] }\end{array}$ \\
\hline Everyday Knowledge & $\begin{array}{l}\text { "I'm going to say pipe B because } \\
\text { when you have a garden } \\
\text { hose and you put your } \\
\text { thumb over the end of the } \\
\text { hose to make it smaller, it } \\
\text { shoots out faster, and } \\
\text { sprays."-Bernie [WP, speed] }\end{array}$ & $\begin{array}{l}\text { "Resistance, is it kind of harder or } \\
\text { easier to move? So if I make an } \\
\text { analogy, it's kind of easier to move } \\
\text { through large openings than it is } \\
\text { through narrow openings. It's just } \\
\text { the common sense. Like if, if you } \\
\text { see it's a bottleneck, right? Like a } \\
\text { traffic, um ... It's a bottleneck. So } \\
\text { the resistance is higher the } \\
\text { narrower the opening is. Um, but } \\
\text { that's just intuition speaking."- } \\
\text { Pat [BV, resistance] }\end{array}$ & $\begin{array}{l}\text { "And so it depends on what kind of } \\
\text { material. So like for instance, if } \\
\text { you pump the same amount of } \\
\text { water through cast iron, which has } \\
\text { a rough internal wall, and you } \\
\text { pump it through PVC, which is } \\
\text { smooth, you'll have less friction } \\
\text { loss. And therefore, less pressure } \\
\text { loss."-Emerson [BV, resistance] }\end{array}$ \\
\hline
\end{tabular}

structures that originate from their learning and work experiences in biology, and they are applying that knowledge to a seemingly nonbiological system. Thus we believe they have framed the WP prompt with a Biological Frame.

We also observed all three physicists using knowledge originating from time spent in their discipline. Throughout the physics responses, we identified instances of explicit use of disci- plinary knowledge like electrical currents, conservation of mass, and shear gradients. The explicit usage of this physics knowledge was apparent through participants' reasoning about the BV prompt, and again when discussing the WP prompt. The following an example shows how Peyton used their disciplinary knowledge to reason about resistance in response to the BV prompt: 
"Viscosity has to do with shear. [clarifying question from T.S.] A shear gradient. Viscosity is a material property that has to do with the shear gradient." [BV, resistance]

One physicist clearly used disciplinary knowledge to connect the BV prompt to current (although they were not specific about what type of current), and then later commented on how this related to current in a circuit, which we take to be an electric circuit.

\begin{abstract}
"So then the flow rate is the same for all three examples because again, the current generally is the same. [and later] So the concept again has to do with compressibility-incompressibility of the fluid. Because it's incompressible and because the current is the same. Uh, at every point along a circuit in a way, you must have the same amount of fluid pass at every, at any given point along this horizontal axis where fluid flow takes place."-Pacey [BV, fluid flow rate]
\end{abstract}

This reliance on discipline-specific knowledge despite being asked about an out-of-discipline prompt suggests physicists have applied a Physical Frame and have activated resources consistent with that frame.

Engineers, like physicists, used different knowledge that sharply contrasts with biologists. We identified multiple instances of engineers introducing concepts or ideas that seemed to originate from their experiences working in their discipline. Notions about mathematical concepts linked to speed and velocity were observed:

"When you ask engineers or physicists, they, speed is, uh, isVelocity is a vector, speed is a, is just kind of a, a scalar product."-Emerson [BV, speed]

Elements of abstraction were also evident, in thinking about the fluid as airflow:

"Yep. It's just that ... Yeah, to a, to an engineer or other people, fluid ... air is a fluid. So it's, it's just a different density, different, it's got different characteristics but it, in many ... The difference between air and water is that air is compressible and that adds some differences in the equations and calculations."-Emerson [WP, fluid flow rate]

And connecting that with other mechanical phenomena, like airplanes flying:

"Well, a lot of times for calculating, uh, the, your ... Bernoulli's principle governs the ... It, it's pretty much the same principle governing why airplanes fly."-Emerson [WP, fluid flow rate]

Engineers and physicists often articulated a specific principle and then followed with an application of that principle to the scenario provided in the prompt. For many of the engineers and physicists, highlighting the fact that the fluids were incompressible and that all matter is contained within the system were important to how they thought about the problem.

"So blood is fairly incompressible, I believe. So since you have the same current left and right ... That means material conver- sation per unit length along the flowing direction, meaning that the speed has to grow when the diameter of the vessel becomes smaller."-Pacey [BV, speed]

"My reason for that. Okay, we have a constant volume. I'm assuming that the blood is basically incompressible as a fluid, or liquid, actually."-Emery [BV, speed]

These observations suggest the engineering experts are not activating a Biological Frame, which is not surprising considering the contextual focus of their specific field. Instead, we see evidence of engineers activating knowledge structures and behaviors similar to those activated by physicists (e.g., Bernoulli's principle, focusing on the physical properties of fluid), suggesting to us the engineering experts have employed a Physical Frame.

\section{Everyday Knowledge as Evidence of Framing}

We also observed participants reasoning using ideas from their everyday experiences rather than disciplinary content knowledge, which collectively formed the theme, Everyday Knowledge. We interpreted this theme as evidence of an interview subject operating within an Everyday Frame.

For example, three biologists-Blake, Bernie, and Blaireach made reference to garden hoses or domestic plumbing in response to the WP prompt (Table 2, Everyday Knowledge, Bernie). In the following example, we see how Blair draws on their everyday experience with garden hoses to generate their predictions:

"Because the constriction, I'm thinking of a water hose and like how much it's gonna shoot out when it comes out. And if you restrict a water hose, this will just... the big one [points at pipe $C$ ] will trickle down, this one [points at pipe B] will shoot out, and this one [points at pipe A] will be in between those two." [WP, speed]

Blair is using their previous experiences with garden hoses to explain how the speed will differ across the three systems, and Blair explicitly states that they are thinking about water moving through hoses. This quote, and others like it, are in response to the WP version of the prompt. Considering the lack of disciplinary knowledge provided in this response and applying our theoretical lens, we would hypothesize that Blair has applied a sort of Everyday Frame, interpreting our prompt in such a way that aligns with, and thus activates, their knowledge and experience with the real-world phenomena they have encountered in everyday life.

Physicists and engineers were less likely to incorporate what we would consider to be everyday knowledge in their responses. We provide two examples in Table 2 (Everyday Knowledge, Pat and Emerson), though these were not nearly as representative of the rest of the explanations generated by physicists and engineers. Similar to what we saw in the biologists' responses, Pat and Emerson provided this kind of real-world knowledge in response to the out-of-context prompt.

\section{Switching Contexts as Evidence of Framing}

At some point during each of their individual interviews, all four biology faculty explicitly articulated thinking and reasoning 
about the cardiovascular system when responding to the WP prompt. These instances when the interview subject explicitly or implicitly recontextualized the prompt were gathered under the theme Switching Context. This theme was then used to determine when individuals moved between frames, for example from the Biological Frame to the Everyday Frame or from the Everyday Frame to the Physical Frame.

Biology faculty used language and concepts that indicated they were thinking about cardiovascular systems, despite responding to questions about water and pipes; biology faculty switched the superficial context of our prompt to align with content from their own discipline. After switching contexts, biology faculty went on to expand the focus of our prompt, introducing additional structures and features that would be relevant to a discussion about cardiovascular physiology. From our theoretical perspective, these observations are consistent with an individual framing the WP prompt as a biology question and, as our framework predicts, activating conceptual and epistemological resources that are consistent with that frame.

At times, biology participants would even call our attention to this framing by explicitly describing how they were relying on their knowledge of cardiovascular physiology. For example:

"Or that how fast blood flows. So when you said speed, the first thing that came to my mind was actually just blood flow. I didn't think about it in any other way besides blood flow."Blake [WP, speed]

Despite being asked about water and pipes, Blake has applied a Biological Frame to our question. Blake's framing may be in response to the underlying conceptual component of our prompt (fluid dynamics). Alternatively, this framing could be in response to a number of other factors that are both known and unknown to the interviewer and the participant. As Gouvea et al., (2019), p. 2 note, framing can be "influenced by both an individual's prior knowledge and experiences and by the physical and social cues presented by a setting." No matter the cause of the framing, we would expect Blake to activate biologyrelated conceptual resources, which in this case would be resources related to cardiovascular flow dynamics. When we look to Blake's earlier explanation to the speed question, we see them focusing on the radius of the pipe and, later, what we believe to be an equation meant to resemble what would be found in an introductory anatomy and physiology textbook:

"But what was most influential, um, was the radius at the end of the pipe on the right-hand side. [clarifying question from T.S.] So, the relationship, um, between radius and speed is ... direct, meaning that although I don't have the equation memorized, flow equals I believe it's the change in pressure times pi times radius to the fourth over I believe it's viscosity times length."

Blake's equation uses language and terms more aligned with those used in cardiovascular physiology than in physics, suggesting Blake has applied a Biological Frame and thus activated a set of locally coherent resources associated with that Biological Frame.

We also observed instances when biology faculty were less explicit about switching contexts:
“Um... I don't really remember capillary physics all that well but I think that pipe B would have the highest speed of water coming out of it, pipe A would have the medium and pipe $\mathrm{C}$ would have the least."-Bailey [WP, speed]

Bailey stated they do not remember capillary physics, a unit unfamiliar to us in either physics or biology courses. This statement suggests to us that Bailey considers the principles of capillary physics, which we believe refers to the biological phenomena that occur in capillaries, useful for answering this question. Thus, Bailey has applied a Biological Frame, not a Physical Frame. By framing this as a biology problem, we would predict Bailey would be inclined to employ conceptual resources associated with biology, thus explaining their mention of capillary physics. However, in this instance, Bailey could not articulate any resources from this frame and seemed to shift frames. Bailey goes on to compare the scenario depicted in our prompt to previous experience handling a syringe. To Bailey, their experience and knowledge gained from using a syringe is relevant and useful for the situation at hand. We propose Bailey appears to adopt an Everyday Frame:

"I couldn't remember how the equations were so I tried to think about what would happen if you just had these from previous experience, so if you try to force a large volume at the same basic pressure through a smaller tube, then like the water would squirt out much farther. Um, so I assume that translated to speed, I'm not super sure. Um... yeah, so that was like pretty much what it was based on."-Bailey [WP, speed]

Compared with the biologists, physicists and engineers were less inclined to explicitly identify when they used their discipline-specific context. Similar to biologists, there were instances when physics and engineering faculty framed the context to be consistent with their own discipline (applying a Physical Frame), when they used words like "pipe" or "water" in their response to a BV prompt:

\footnotetext{
"Well, you've got the same volume or flow rate, so many gallons per minute or whatever, right? And you've got [the] same pressure, pressure hasn't changed. So you've got a smaller area, right? So, flow in pipes is related to... if you've got the same flow coming into all of these and you got the same flow going through here [pointing to handout], the only way you can make up for it is to increase the velocity."-Emerson [BV, speed]
}

While this framing is not as explicit as the framing observed in biology faculty responses, it represents a shift away from a biological context. Contexts of water flowing through pipes are canonical in physics and engineering textbooks, and using these words is evidence for activating a Physical Frame. In doing so, faculty within this frame are expected to call on resources that exist within their Physical Frame. This is seen in Emery's response calling on the continuity equation:

"I'm assuming that the blood is basically incompressible as a fluid, or liquid, actually. And so $Q$ is equal to VA, velocity times cross-sectional area. $V$ is then, uh, $Q$ divided by $A$. And the smallest $A$ produces the, um, biggest velocity, for $Q$ being a constant." [BV, speed] 
In this case, Emery's use of the continuity equation likely stems from the activation of conceptual and epistemological resources tied to their Physical Frame. Again, these cases were neither as explicit or as frequent as those observed with biology faculty, but they are framed distinctly from how the prompt is presented.

Physicists and engineers often removed the biological context for the BV version of our prompt, as demonstrated by Peyton:

\begin{abstract}
“The fluid's moving, so it's not a simple hydraulic kind of problem where I have a weight on one side and it pushes down on a cross-sectional area and that pushes up on a different cross-sectional area and then I make a ratio and get my forces that way." [BV, pressure]
\end{abstract}

Here, Peyton refers to the blood as "fluid" and references it as "not a simple hydraulic" problem. Instead of adding additional context, like we saw in the biologists' responses, we see Peyton removing context. Although it is a different strategy when compared with the previous example of framing the BV prompt to be about water in pipes, abstracting the problem to a generic fluid is also a common practice, especially in physics. We would expect to see activation of resources from a Physical Frame, like the relationship of pressure, force, and area:

"Pressure is force divided by area."-Peyton [BV, pressure]

We observed all experts switching contexts, whether subconsciously or not, when responding to the out-of-discipline prompt. Based on our theoretical lens of framing and resources, these context switches revealed how experts were framing our interview prompt. In some cases, these frames were made explicit, with experts either directly calling attention to alternative content (e.g., Blake with a Biological Frame) or by incorporating new structures or terms that pertain to alternative content (e.g., Bailey with a Biological Frame). Alternatively, there were many cases in which expert frames were more subtle. In these instances, expert framing was made apparent by the use of cognitive structures pertinent primarily to the alternative content (e.g., Emery with a Physical Frame).

\section{A Note on Resistance}

We found that resistance was a productive idea for biologists but less so for physicists. Some biology faculty used the concept of resistance when reasoning about questions that did not ask about it. Blake was especially reliant on the concept of resistance, and we see them explicitly make use of this idea early on during the interview:

\begin{abstract}
"Okay. So the pressure does depend on volume, but it also depends on resistance, um, and resistance does depend on the radius of these pipes. And I know that the, um, the bigger the radius, the less resistance there will be. And the less resistance there is, the less, um, pressure there is on the wall of the pipe." [WP, pressure]
\end{abstract}

Other biology faculty were less inclined to use the term "resistance" but provided reasoning that suggested they were using the principle of resistance without explicitly making mention of the term. Bernie, for example, focused their reasoning on the diameter of the tube to generate a prediction based on their experience with vasoconstriction and, ultimately, resistance:

"Alright. So it's flowing in at the same rate in each pipe, and is at the same pressure until it reaches the bottleneck, and then we have different diameters. I know that ... I think all that matters ... I know with like blood vessels, when you vasoconstrict that can raise blood pressure, for sure. I'm going to say pipe B because when you have a garden hose and you put your thumb over the end of the hose to make it smaller, it shoots out faster, and sprays." [WP, speed]

This reliance on resistance is not surprising. Resistance is an important concept for biologists (Redish and Cooke, 2013), and HA\&P textbooks typically do not consider fluid flow in the absence of resistance due to its importance on the cardiovascular system.

In contrast to biologists, physics faculty did not incorporate resistive effects and did not use the term "resistance" when answering the first three items. When asked about resistance in the fourth question, all physics faculty were completely unfamiliar with the term in the context of fluid flow and asked for clarification about the definition of resistance. In addition, physics faculty struggled to offer a definition of resistance in this context:

"I would guess you're getting somehow at some kind of weird conceptual idea of what viscosity might be like in it? Either that or how hard it would be for the fluid to go through the tube. Both seem kind of ill-designed."-Peyton [BV, resistance]

The physics faculty were still not comfortable answering questions about resistance after being provided with a working definition from biology. Physics faculty found the biological definition of resistance to be problematic and, at times, voiced concern with how biologists use the term "resistance" in the context of fluid dynamics:

T.S.: "Did you think that [resistance] was a useful question?" Peyton: "Nope, I think it's a bad idea."

Peyton's comments were one of the more pointed examples of a common perspective-physicists were dissatisfied with the way resistance is represented in the introductory physiology curriculum. This difference in opinion leads us to presume physicists would not teach resistance in a way that resembles how a biologist might teach resistance and fluid dynamics.

This contrasting opinion aligns with the work of Redish and Cooke (2013) and exemplifies the epistemological differences found between physics and biology. The underlying concepts that give rise to a biologist's view of resistance are discussed in introductory physics courses (i.e., length, diameter, viscosity), though never in association with the term resistance:

"Viscosity is a material property so it's the same for the material, but the, the loss or the hardness of pushing the fluid has to do with how far the walls are apart."-Peyton [BV, resistance]

It is interesting to note that physicists regularly use the term "resistance" in the context of electric circuits. We observed all 
three participants incorporating ideas from circuits when explaining their reasoning on the resistance questions:

T.S.: "Is that [resistance] a familiar concept at all to you?" Pat: "No, but I can make an analogy with, um, resistance in electric circuits."

Finally, engineers appeared to have more varied perspectives on the concept of resistance compared with physicists. Similar to physicists, Emerson was somewhat uncomfortable with describing resistance as a force. Conversely, when asked to define resistance, Emery offered a description similar to what we might expect from a biologist:

"Opposition to the flow. And that which I would-is partially defined as the change in pressure between the two reference points."-Emery [BV, resistance]

\section{General Discussion}

To our knowledge, there are few instances of the framing and resources framework being used to explain expert reasoning across disciplines. We believe this theoretical framework is well suited for describing expert reasoning, as it accommodates both the formal disciplinary knowledge that an expert acquires through training, as well as the more informal, intuitive knowledge that an expert would acquire experiencing the natural world (diSessa, 1988). The framing and resources framework respects disciplinary norms, accommodates epistemological differences across distinct domains, and emphasizes and elucidates the impact context has on reasoning. Without this theoretical frame, we may recognize a difference in expert reasoning, but we would be unable to understand or explain why that difference existed.

\section{Disciplinary Expertise, Context, and Framing}

We asked faculty from biology, physics, and engineering to reason about the crosscutting concept of fluid dynamics in two contextual settings, blood/vessels and water/pipes. We found little disagreement within and across disciplines in response to the speed and resistance questions. We observed a great deal of variation among biologists in response to the fluid flow rate question, and those responses were quite different from the responses provided by the physicists and engineers. There was very little disagreement among biologists in response to the pressure question, though we did observe considerable disagreement within physicists and engineers for this specific item. One might assert that one disciplinary group of experts is "wrong" and another is "right."

We leverage the framing and resources theoretical framework to contest this perspective. We observed experts using knowledge and behaviors from their own disciplines when presented with the out-of-context prompt. In accordance with the framing and resources theoretical framework, we interpret this finding as framing these problems in distinctly different ways, thus activating different suites of conceptual and epistemological resources. In turn, this differential resource activation could result in our experts attending to different aspects of our prompt, employing different reasoning strategies, or even striving for different reasoning outcomes, all of which could ultimately lead to two different, yet equally appropriate, ranking responses.
We would also argue such a traditional perspective of accuracy fails to recognize the inherent and important cultural and epistemological differences of the disciplines. Biology and physics are different disciplines, with distinct ways of knowing the natural world. Redish and Cooke (2013) argue physicists find value in simplified or abstracted systems models and are often more interested in how outside forces impact the system of interest. Redish and Cooke contrast those values and behaviors with what they suggest to be true of biologists-embracing complexity, working to understand the mechanisms underpinning complex living systems.

We found evidence to empirically support the perspectives of Redish and Cooke (2013), as these cultural and epistemological differences were present throughout our interview data. For example, biologists expanded the focus of our prompt to more authentically discuss cardiovascular physiology, even when reasoning about a WP question. Physicists focused more deeply on the constraints of the system and would often abstract the biology content out of the BV prompt. Regardless of prompt version, physicists had a tendency to simplify the focus of our prompt, whereas biologists had a tendency to make it more complex. We found engineers had behaviors somewhat intermediate to both biologists and physicists. Much like physicists, engineers emphasized the importance of the constraints on the system (i.e., conservation of matter); however, they were more conservative in the ways they likened water to blood when reasoning across our prompts.

These observations suggest experts applied a familiar disciplinary frame (i.e., a Biological Frame or Physical Frame) to the out-of-context version of our prompt and activated conceptual and epistemological resources consistent with that frame. Further, our evidence suggests the application of these frames could activate conceptual and epistemological resources that give rise to the cultural and epistemological characteristics inherent to biology or physics introduced by Redish and Cooke (2013), regardless of whether an expert is actually presented with a biology or physics problem.

We saw little evidence that faculty rankings were affected by the surface contexts of our prompts (Table 1). Most faculty used similar reasoning strategies and demonstrated similar behaviors compared with their disciplinary counterparts and often came to the same conclusions in both prompt contexts (BV and WP). Instead, our evidence suggests it was the underlying content of our prompt (fundamental fluid dynamics) that impacted expert framing and resulting reasoning.

Hammer and colleagues (2005) offer that the recurring activation of a particular frame, and the resulting resources, could result in that frame being more permanently established as a cognitive unit. Because all of our experts have extensive training and experience working in their respective disciplines, it is possible they have well-established frames (or even cognitive units), and it could be the case that the underlying content of our prompt (the focus on a fluid dynamics) cued them to activate those established frames despite the surface context being outside of their discipline. These experts have gone through years of training to think like experts in their fields, and that training has likely reinforced framing behaviors that are productive in their respective fields. 


\section{Implications for Research}

In the present research, we propose several frames that experts may adopt when solving foundational fluid dynamics problems. In doing so, we also provide empirical evidence for the disciplinary epistemological differences identified by Redish and Cooke (2013) - in essence, how biologists reason about phenomena in fundamentally different ways from physicists. We predict these frames are applicable to many crosscutting concepts in STEM-for example, energy or movement of gasesand that additional frames are likely to emerge from chemistry, biochemistry, and mathematics. The theoretical framework of framing and resources will support further research into disciplinary epistemologies.

Further, differences in expert and novice reasoning (Chi et al., 1981) suggest students may adopt different frames in response to item context features. In our study, experts largely ignored item context, framing their reasoning in response to the underlying concept of fluid dynamics. However, as demonstrated in sorting tasks (Chi et al., 1981; Smith et al., 2013), students often focus on item context features. Thus, we might predict that item surface features, along with other context effects, would activate different frames for students. Again, we suggest the theoretical framework of framing and resources as an essential tool to further exploration of student framing of crosscutting problems and student reasoning.

\section{Implications for Instruction}

The differences in ranking responses we observed across disciplines reflect experts' extensive disciplinary experiences, which have shaped them into expert thinkers. Taking into account differing epistemological cultures and the potentially disparate conceptual frames and resources routinely activated by our experts, it is appropriate that faculty from different disciplines would frame a problem within their own disciplines, making disciplinary assumptions about the problem, and thereby reach different conclusions when reasoning about a crosscutting concept like fluid flow. When biologists reason about a crosscutting problem using a biological frame and the resulting conceptual resources, they make sense of the system and generate predictions in a way that is productive in the field of biology. It is possible their reasoning and resulting predictions may be seen as incomplete in the eyes of a physicist, but what is productive in one discipline will not necessarily be seen as productive or sufficient in another due to the epistemological differences between disciplines.

While it may be appropriate that experts frame problems on crosscutting concepts differently, this disciplinary framing might flow into instruction, which could impede students' transfer of crosscutting ideas. Knowledge can be tightly bound to context, particularly when learners are taught in a single context (Bjork and Richardson-Klavehn, 1989). This is often the case in undergraduate STEM classrooms-students are taught about a concept like fluid flow in the context of physics or biology and so learn to associate specific ideas with those classes and associated norms. Students then struggle to see the connection between these classes and may not be able to recognize the epistemological differences between the disciplines, and thus are unable to reconcile seemingly competing instruction. Instruction that helps learners abstract concepts and principles, however, supports transfer and may help students bridge the gaps between their introductory STEM courses (Gick and
Holyoak, 1983). For this to occur, instructors need to develop a working understanding of how these crosscutting concepts are used and taught in different disciplines and an ability to use cases and examples that bridge disciplines.

In the case of fluid dynamics, many students enrolled in anatomy and physiology at our institution are required to complete the introductory algebra-based physics series, and many students enroll in these courses simultaneously. Based on the findings from our study, it is likely that these students will receive distinct physics and biology instruction and will struggle to see fluid flow in physics as the same concept in anatomy and physiology. Instructional practices currently do not support that transfer of understanding.

We encourage instructors, especially those teaching introductory courses, to connect with faculty across STEM departments to discuss the crosscutting concepts they teach in their courses, in addition to identifying pedagogical decisions that support students' development of a coherent understanding of crosscutting concepts. Further, we recommend instructors observe STEM classes and read discipline-based education research outside of their own disciplines to gain awareness and appreciation of the cultural and epistemological differences present across disciplines. These immersive experiences may reveal new insights that could inform how we teach crosscutting concepts in our classrooms and, ultimately, better support student transfer.

As a community of scientists, we must engage in the difficult process of exploring and comparing disciplinary expectations that surround crosscutting concepts in STEM. Engaging in such dialogue will help faculty create instruction that bridges disciplines and helps students transfer knowledge across domains. Transfer between courses is hard enough (NRC, 2000). Students need support to connect and organize the bits and pieces of crosscutting concepts they collect during their academic careers.

\section{Limitations}

One of the major challenges in studying student or expert understanding of cross-cutting concepts, like fluid dynamics, is in developing assessment prompts that are authentic to multiple disciplines (Reinholz et al., 2019). An authentic problem is contextualized, a process made more difficult when the context must be relevant to multiple disciplines. In addition, the problem must also be broad enough to accommodate different disciplinary approaches and knowledge. A typical approach to this contextualization challenge is to make the surface features of a problem relate to a second domain while the underlying or deep features remain discipline specific. For example, a problem that asks students to find the maximum height of a frog that jumped with a certain initial velocity does not automatically make the problem cross-disciplinary; such an approach has been likened to "pouring salsa over a meatloaf and calling it a Mexican dish" (Meredith and Bolker, 2012, p. 914).

We found it essential to work collaboratively on prompt creation. More than writing a prompt, this process involved an extended, and at times contentious, discussion of fluid dynamics as it was represented and taught in each discipline. We worked to build a shared language surrounding fluid dynamics and to identify concepts that were core to both biology and physics. Thus, these conversations helped us identify the essential disciplinary elements that became the core of our prompt. 


\section{CONCLUSION}

The framing and resources framework was a productive approach to supporting our exploration of expert reasoning about a cross-cutting concept. We found that experts, despite being asked to reason about a context outside their own disciplines, framed the problems in ways that aligned with their disciplinary backgrounds and thus activated conceptual resources connected to that frame. Through this framing, we found that experts differed in their epistemological problem-solving behaviors and in the importance ascribed to resistance, a productive concept in physiology. By extension, it is plausible that experts then teach in ways that align with their disciplinary framing and subsequently reinforce disciplinary boundaries already perceived by STEM students.

\section{ACKNOWLEDGMENTS}

We thank the faculty who participated in our study, including those who piloted our prompt and provided feedback during development. Without their participation, this study could not have been conducted. We thank members of the Momsen lab for many valuable discussions. We also thank the University of Maryland Physics Education Research Group, especially Edward (Joe) Redish, for their insightful discussions and feedback on this project. This study was supported by the North Dakota State University Graduate School through the STEM Education PhD Fellowship and the North Dakota Experimental Program to Stimulate Competitive Research Infrastructure Improvement Program-Doctoral Dissertation Assistantship.

\section{REFERENCES}

Berland, L. K., \& Hammer, D. (2012). Framing for scientific argumentation Journal of Research in Science Teaching, 49(1), 68-94.

Bing, T. J., \& Redish, E. F. (2008). Symbolic manipulators affect mathematical mindsets. American Journal of Physics, 76(4), 418-424.

Bjork, R. A., \& Richardson-Klavehn, A. (1989). On the puzzling relationship between environmental context and human memory. In Izawa, C. (Ed.), Current issues in cognitive processes: The Tulane Flowerree Symposium on Cognition (pp. 313-344). Laurence Erlbaum Associates, Inc.

Bodner, G. M., \& Herron, J. D. (2002). Problem-solving in chemistry. In Chemical education: Towards research-based practice. Dordrecht, Netherlands: Springer

Braun, V., \& Clarke, V. (2006). Using thematic analysis in psychology. Qualitative Research in Psychology, 3(2), 77-101.

Camacho, M., \& Good, R. (1989). Problem solving and chemical equilibrium: Successful versus unsuccessful performance. Journal of Research in Science Teaching, 26(3), 251-272.

Carnegie Classification of Institutions of Higher Education (n.d.). About Carnegie Classification. Retrieved November 11, 2019, from http:// carnegieclassifications.iu.edu

Chi, M. T., Feltovich, P. J., \& Glaser, R. (1981). Categorization and representation of physics problems by experts and novices. Cognitive Science, 5(2), 121-152.

diSessa, A. A. (1988). Knowledge in pieces. In Forman, G., \& Putall, P. (Eds.), Constructivism in the computer age (pp. 49-70). Hillsdale, NJ: Erlbaum.

diSessa, A. A. (1993). Toward an epistemology of physics. Cognition and Instruction, 10(2/3), 105-225.

diSessa, A. A., Gillespie, N. M., \& Esterly, J. B. (2004). Coherence versus fragmentation in the development of the concept of force. Cognitive Science, 28(6), 843-900.

Elby, A., \& Hammer, D. (2010). Epistemological resources and framing: A cognitive framework for helping teachers interpret and respond to their students' epistemologies. In Bendixon, L. D., \& Feucht, F. C. (Eds.), Personal epistemology in the classroom: Theory, research, and implications for practice (pp. 409-434). Cambridge, England: Cambridge University Press.
Farlow, B., Vega, M., Loverude, M. E., \& Christensen, W. M. (2019). Mapping activation of resources among upper division physics students in non-Cartesian coordinate systems: A case study. Physical Review Physics Education Research, 15(2), 020125.

Gick, M. L., \& Holyoak, K. J. (1983). Schema induction and analogical transfer Cognitive Psychology, 15(1), 1-38.

Giordano, N. J. (2013). College physics: Reasoning and relationships (2nd ed.). Pacific Grove, CA: Brooks/Cole Cengage Learning.

Goodhew, L. M., Robertson, A. D., Heron, P. R., \& Scherr, R. E. (2019). Student conceptual resources for understanding mechanical wave propagation. Physical Review Physics Education Research, 15(2), 020127.

Gouvea, J., Sawtelle, V., \& Nair, A. (2019). Epistemological progress in physics and its impact on biology. Physical Review Physics Education Research, 15(1), 010107

Gouvea, J. S., \& Simon, M. R. (2018). Challenging cognitive construals: A dynamic alternative to stable misconceptions. CBE-Life Sciences Education, 17(2), ar34

Hammer, D. (1996). Misconceptions or p-prims: How may alternative perspectives of cognitive structure influence instructional perceptions and intentions? Journal of the Learning Sciences, 5(2), 97-127.

Hammer, D. (2000). Student resources for learning introductory physics. American Journal of Physics, 68(S1), S52-S59.

Hammer, D., \& Elby, A. (2003). Tapping epistemological resources for learning physics. Journal of the Learning Sciences, 12(1), 53-90.

Hammer, D., Elby, A., Scherr, R. E., \& Redish, E. F. (2005). Resources, framing, and transfer. Transfer of Learning from a Modern Multidisciplinary Perspective, 89-120.

Heredia, S., Furtak, E. M., \& Morrison, D. (2012). How organisms used to frame natural selection items influence student response choices. In Proceedings of the National Association for Research in Science Teaching (NARST) Annual Conference, held April 2012 in Indianapolis, IN.

Human Anatomy and Physiology Society. (2019). Anatomy and physiology learning objectives. Retrieved June 16, 2020, from www.hapsweb.org/ page/Learning_Outcomes.

Knight, R. D., Jones, B., \& Field, S. (2010). College physics: A strategic approach (2nd ed.). San Francisco: Pearson/Benjamin Cummings.

Lising, L., \& Elby, A. (2005). The impact of epistemology on learning: A case study from introductory physics. American Journal of Physics, 73(4), $372-382$.

Loverude, M. (2015). Identifying student resources in reasoning about entropy and the approach to thermal equilibrium. Physical Review Special Topics-Physics Education Research, 11(2), 020118

Martini, F. H., Nath, J. L., \& Bartholomew, E. F. (2015). Fundamentals of anatomy and physiology (10th ed.). San Francisco: Pearson/Benjamin Cummings.

Maskiewicz, A. C., \& Lineback, J. E. (2013). Misconceptions are "so yesterday!" CBE-Life Sciences Education, 12(3), 352-356.

McDonald, J. (2015). The Next Generation Science Standards: Impact on college science teaching. Journal of College Science Teaching, 45(1), 13.

Meredith, D. C., \& Bolker, J. A. (2012). Rounding off the cow: Challenges and successes in an interdisciplinary physics course for life science students. American Journal of Physics, 80, 913-922.

National Research Council (NRC). (2000). How people learn (expanded ed.) Washington, DC: National Academies Press.

NRC. (2012). A framework for $K-12$ science education: Practices, crosscutting concepts, and core ideas. Washington, DC: National Academies Press.

Nehm, R. H., \& Ha, M. (2011). Item feature effects in evolution assessment. Journal of Research in Science Teaching, 48(3), 237-256.

Redish, E. F., \& Cooke, T. J. (2013). Learning each other's ropes: Negotiating interdisciplinary authenticity. CBE-Life Sciences Education, 12(2), 175-186.

Redish, E. F., \& Kuo, E. (2015). Language of physics, language of math: Disciplinary culture and dynamic epistemology. Science \& Education, 24, 561-590.

Reinholz, D., Slominski, T., French, T. A., Pazicni, S., Rasmussen, C., \& McCoy, B. (2019). Good problems within and across disciplines. Journal of Research in STEM Education, 4(1), 37-53.

Sabella, M. S., \& Redish, E. F. (2007). Knowledge organization and activation in physics problem solving. American Journal of Physics, 75(11), $1017-$ 1029. 
Simmons, P. E., \& Lunetta, V. N. (1993). Problem-solving behaviors during a genetics computer simulation: Beyond the expert/novice dichotomy. Journal of Research in Science Teaching, 30(2), 153173.

Silverthorn, D. U. (2009). Human physiology: An integrated approach (4th ed.). San Francisco: Pearson/Benjamin Cummings.

Slominski, T. N., Momsen, J. L., \& Montplaisir, L. M. (2017). Drawing on student knowledge of neuroanatomy and neurophysiology. Advances in Physiology Education, 41(2), 212-221.

Smith, J. I., Combs, E. D., Nagami, P. H., Alto, V. M., Goh, H. G., Gourdet, M. A. ... \& Tanner, K. D. (2013). Development of the biology card sorting task to measure conceptual expertise in biology. CBE-Life Sciences Education, 12(4), 628-644.

Smith, J. P., diSessa, A. A., \& Roschelle, J. (1994). Misconceptions reconceived: A constructivist analysis of knowledge in transition. Journal of the Learning Sciences, 3(2), 115-163.

Smith, M. U., \& Good, R. (1984). Problem solving and classical genetics: Successful versus unsuccessful performance. Journal of Research in Science Teaching, 21(9), 895-912.

Wittmann, M. C., \& Black, K. E. (2015). Mathematical actions as procedural resources: An example from the separation of variables. Physical Review Special Topics-Physics Education Research, 11(2), 020114. 